\title{
Histomorphometry of the optic disc in highly myopic eyes with absolute secondary angle closure glaucoma
}

\author{
Albert Dichtl, Jost B Jonas, Gottfried O H Naumann
}

\begin{abstract}
Aim-To evaluate histomorphometrically the optic nerve head in highly myopic eyes with absolute secondary angle closure glaucoma.

Methods-Optic disc sections of 16 highly myopic eyes with an axial length of more than $26 \mathrm{~mm}$ and of 19 eyes with an axial length of less than $26 \mathrm{~mm}$ were histomorphometrically evaluated. All eyes had been enucleated due to painful absolute secondary angle closure glaucoma.

Results-In the highly myopic eyes compared with the non-highly myopic eyes, mean optic disc diameter was significantly larger (mean 2.33 (SD 0.55 ) $\mathrm{mm}$ versus $1.77(0.50) \mathrm{mm} ; \mathrm{p}=0.01)$, and the optic cup was significantly shallower (optic cup depth $0.34(0.29) \mathrm{mm}$ versus $0.63(0.23)$ $\mathrm{mm} ; \mathrm{p}=0.03)$. The peripapillary scleral ring was significantly broader $(0.58(0.65)$ mm versus $0.08(0.06) \mathrm{mm} ; p=0.001)$, and the $\beta$ zone $(0.83(0.74) \mathrm{mm}$ versus 0.28 $(0.25) \mathrm{mm} ; \mathrm{p}=0.006)$ of the parapapillary chorioretinal atrophy was significantly larger in the highly myopic eyes.

Conclusions-The results of the present study agree with biomorphometric data of the optic nerve head in highly myopic eyes with glaucoma. In the highly myopic group, a markedly enlarged peripapillary scleral ring characterised by absence of Bruch's membrane and choriocapillaris contributes in addition to $\alpha$ and $\beta$ zone to the parapapillary atrophy.

(Br F Ophthalmol 1998;82:286-289)
\end{abstract}

Remarkable differences exist in the optic disc morphology between glaucomatous eyes with high myopia and glaucomatous eyes with normal axial length. Clinical studies have shown that highly myopic eyes compared with emmetropic eyes have an unusually large and abnormally configured optic nerve head with shallow disc cupping and an unusually large parapapillary atrophy. ${ }^{1-3}$ As histomorphometric correlations of these clinical findings in highly myopic eyes are scarce or lacking, ${ }^{4-7}$ the aim of the present investigation was to identify and measure the histological correlates of the ophthalmoscopic features of the optic disc in glaucomatous eyes with high myopia and to describe differences between these eyes and glaucomatous eyes without high myopia.

\section{Material and methods}

The study included 35 histological sections of 35 globes enucleated due to painful absolute secondary angle closure glaucoma. Various reasons such as perforating corneal injuries had been responsible for the closure of the angle. Medical and surgical therapy such as cyclocryocoagulation did not succeed in reducing intraocular pressure or pain. Posterior to the ora serrata, no surgical intervention had been undertaken. In all sections the retina was attached or only artificially detached.

The eyes were divided in two groups according to axial length. Sixteen eyes had an axial length of more than $26 \mathrm{~mm}$ and were considered to be highly myopic; 19 eyes with an axial length of less than $26 \mathrm{~mm}$ served as a control group (Table 1).

According to the selection criteria mean axial length and mean horizontal and vertical globe diameters were significantly $(\mathrm{p}<0.001$; Mann-Whitney test) larger in the highly myopic group than in the non-highly myopic group (Table 1). The groups did not vary significantly in age $(p=0.33)$ and sex (Table 1$)$.

All globes were fixed immediately after enucleation in a solution of $4 \%$ formaldehyde $/ 1 \%$ glutaraldehyde. All globes were opened in an anteroposterior direction. Histological sections $8 \mu \mathrm{m}$ in thickness going through the pupil and the optic disc were prepared in a routine manner, embedded in paraffin, and stained by the periodic acid Schiff method. The slide which contained the most central part of the optic disc was selected for further evaluation. Both groups contained horizontal and vertical sections in a similar distribution (Table 1).

Using a microscope and a planimeter, the illuminated cursor of which was mirrored into the microscope, we evaluated on every histological slide the diameter of the optic disc defined as distance between the two inner margins of the sclera, and the depth of the optic cup measured between the inner surface of the lamina cribrosa and three reference lines at the level of the uveoscleral interface, Bruch's membrane, and the retinal surface, respectively. In the parapapillary region, we determined the width of the peripapillary scleral ring defined as distance between the optic disc border and the beginning of Bruch's membrane; the length of zone " $B$ " defined as Bruch's membrane bared of retinal pigment epithelium cells; and the length of zone " $A$ " defined as Bruch's membrane covered with irregularly pigmented retinal pigment epithelium cells. The method has already been
Accepted for publication 10 October 1997 
Table 1 Composition of the study groups (mean (SD))

\begin{tabular}{llll}
\hline & $\begin{array}{l}\text { Highly myopic } \\
\text { group }\end{array}$ & $\begin{array}{l}\text { Non-highly myopic } \\
\text { group }\end{array}$ & p Value $^{\star}$ \\
\hline Number & 16 & 19 & \\
Age (years) & $65.3(15.0)$ & $70.5(13.6)$ & $0.33(\mathrm{NS})$ \\
Sex (M/F) & $6 / 9$ & $8 / 11$ & \\
Horizontal/vertical sections & $9 / 7$ & $9 / 10$ & $<0.0001$ \\
Axial length (mm) & $30.4(2.6)$ & $23.6(0.9)$ & $<0.0001$ \\
Horizontal diameter (mm) & $26.0(1.4)$ & $23.1(1.1)$ & $<0.0005$ \\
Vertical diameter (mm) & $25.3(1.6)$ & $23.2(1.2)$ & \\
\hline
\end{tabular}

$\star$ Significance of differences between the two groups; NS = not statistically significant).

explained in detail. ${ }^{8}$ The study design was unmasked with the examiner knowing to which group the histological sections were assigned. To determine the reproducibility, measurements of zone $\mathrm{B}$ of the parapapillary atrophy were repeated five times in randomly selected sections of five eyes of the highly myopic group and in randomly selected sections of five eyes of the non-highly myopic group.

\section{Results}

In the highly myopic eyes compared with the non-highly myopic eyes, the optic disc diameter was significantly larger $(p=0.01$; MannWhitney test) and the optic cup depth measured as distance to the reference level at the retinal surface was significantly $(p=0.03)$ more shallow (Table 2). Taking into account all measured sections, the optic disc diameter was significantly $(p=0.004)$ correlated with the axial length of the globes (Pearson's correlation coefficient $R=0.51$ ). In the parapapillary region, the peripapillary scleral ring was significantly $(p=0.001)$ broader and zone $B$ was significantly $(p=0.006)$ larger in the highly myopic group than in the non-highly myopic group (Table 2). The values for zone A did not differ significantly. Taking into account the larger optic disc diameter and the larger width of the scleral ring and zone B in the highly myopic group compared with the non-highly myopic group, area of zone A calculated as width of zone $\mathrm{A} \times$ circumference of the outer border of zone B was markedly larger in the highly myopic group than in the non-highly myopic group (Figs 1-4). The coefficient of reproducibility of the measurements was $10.8 \%$, determined as mean of the standard deviation divided by the mean of the means.

Table 2 Histomorphometric measurements of the highly myopic group (eyes with absolute secondary angle closure glaucoma and an axial length $>26 \mathrm{~mm}$ ) compared with the non-highly myopic group (eyes with absolute secondary angle closure glaucoma and an axial length $<26 \mathrm{~mm}$ ) (mean $(S D)$

\begin{tabular}{llll}
\hline & $\begin{array}{l}\text { Highly myopic } \\
\text { group }\end{array}$ & $\begin{array}{l}\text { Non-highly myopic } \\
\text { group }\end{array}$ & p Value* \\
\hline $\begin{array}{l}\text { Intrapapillary region: } \\
\text { Optic disc diameter (mm) }\end{array}$ & $2.33(0.55)$ & $1.77(0.50)$ & 0.01 \\
$\begin{array}{l}\text { Optic cup depth (mm) measured: } \\
\text { From the uveoscleral interface }\end{array}$ & $0.20(0.19)$ & $0.34(0.21)$ & 0.09 \\
$\quad$ From Bruch's membrane & $0.24(0.20)$ & $0.41(0.21)$ & 0.08 \\
$\quad$ From retinal surface & $0.34(0.29)$ & $0.63(0.23)$ & 0.03 \\
$\begin{array}{l}\text { Parapapillary region: } \\
\quad \text { Width (mm) of: }\end{array}$ & & & \\
$\quad \begin{array}{l}\text { Peripapillary scleral ring } \\
\text { Zone "B" }\end{array}$ & $0.58(0.65$ & $0.08(0.06$ & 0.001 \\
Zone "A" & $0.83(0.74)$ & $0.28(0.25)$ & 0.006 \\
\hline
\end{tabular}

^Significance of differences between the two groups.

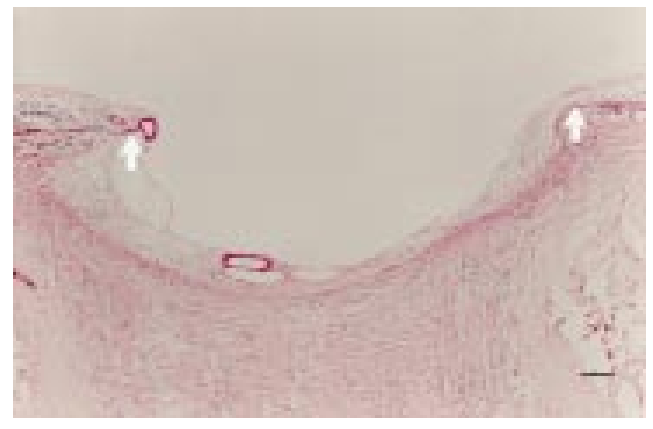

Figure 1 Microphotograph of an optic nerve head cross section of an eye with absolute glaucoma and normal axial length of $24 \mathrm{~mm}$. Note the deep excavation. White arrows indicate the ends of Bruch's membrane. (Magnification as indicated in the right lower corner: black bar $100 \mu \mathrm{m}$; staining, periodic acid Schiff.)

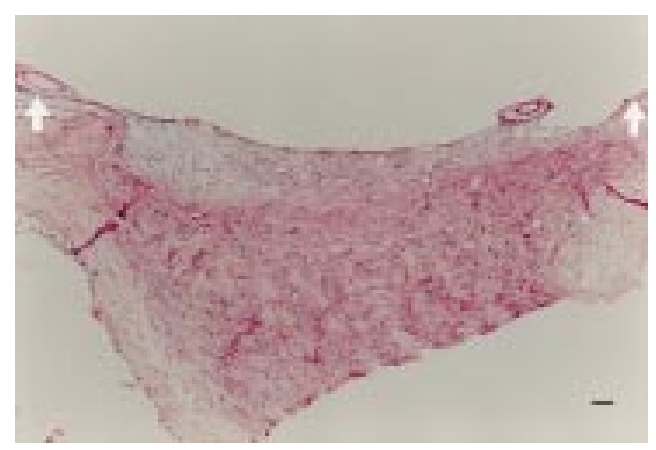

Figure 2 Microphotograph of an optic nerve head cross section of a highly myopic eye with absolute glaucoma (axial length $30 \mathrm{~mm}$ ). It shows a shallow excavation. White arrows indicate the retinochoroidal interface. (Magnification as indicated in the right lower corner: black bar $100 \mu \mathrm{m}$; staining, periodic acid Schiff.)

\section{Discussion}

The histomorphometric results demonstrate abnormally large optic discs in glaucomatous eyes with high myopia. They confirm intravital measurements of the optic disc on fundus photographs. ${ }^{1-3}$ Abnormally large optic discs or "macrodiscs" can be differentiated into primary macrodiscs that are present at birth or in the early years of life, and secondary macrodiscs that are acquired. ${ }^{10}$ The large optic discs in highly myopic eyes are presumably secondary macrodiscs, since in these eyes the optic disc size is positively correlated with the myopic refractive error. ${ }^{2}$ This is confirmed by the correlation between axial length and optic disc diameter in the present study. It suggests that optic discs in highly myopic eyes enlarge due to a myopic stretching of the globe. This stands in contrast with eyes with slight hyperopia or myopia where the optic disc size is independent of the refractive error. ${ }^{11}$

The optic cup as measured histomorphometrically in this study was significantly more shallow in the highly myopic eyes than in the non-highly myopic eyes. It agrees with biomorphometric data of an unusually shallow disc cupping in highly myopic eyes with glaucoma. ${ }^{13}$

In the parapapillary area of the highly myopic eyes, three regions could be differentiated histomophometrically.

(1) The region at the border of the optic nerve scleral canal was formed by the inner surface of the peripapillary scleral ring covered 


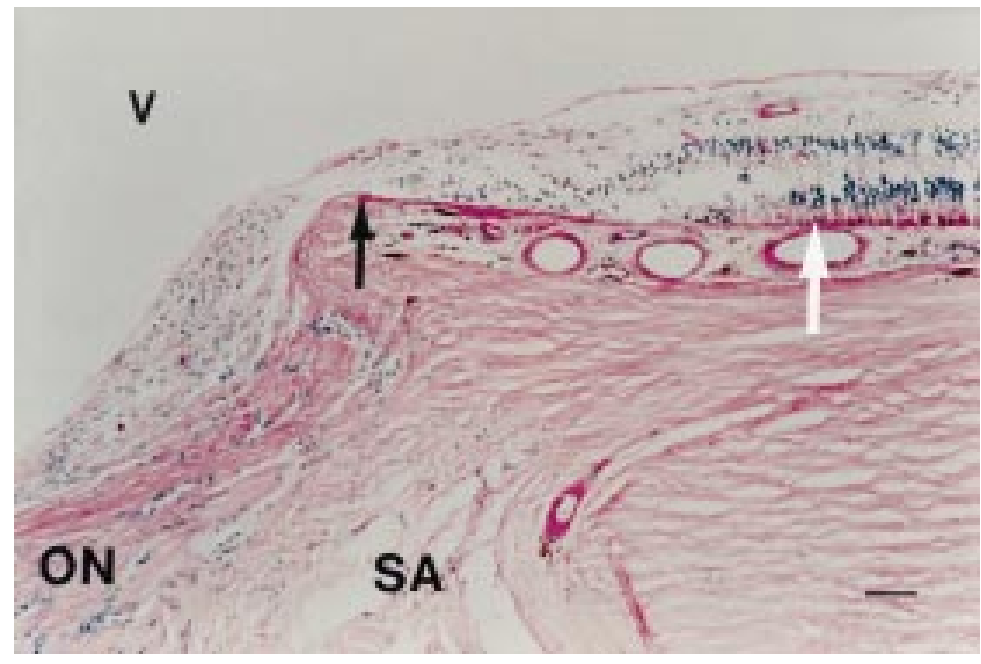

Figure 3 Microphotograph of the parapapillary region of an eye with absolute glaucoma and normal axial length (same eye as Fig 1). The black arrow points to the edge of Bruch's membrane and the white arrow indicates the beginning of the retinal pigment epithelium. The scleral ring is located left of the black arrow, zone $B$ is between the two arrows and zone $A$ is on the right of the white arrow. (ON= optic nerve, $S A=$ subarachnoid space, $V=$ vitreous; magnification as indicated in the right lower corner: black bar $50 \mu \mathrm{m}$; staining, periodic acid Schiff.)

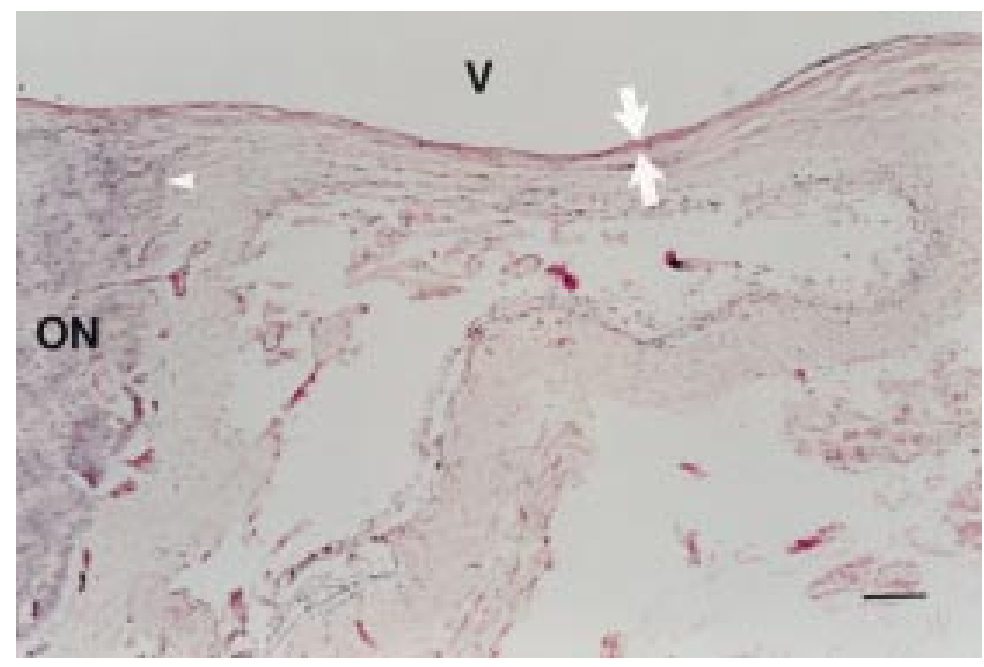

Figure 4 Microphotograph of the parapapillary region of a highly myopic eye with absolute glaucoma (axial length $34 \mathrm{~mm}$ ). The white arrows indicate the retinal remnants. Bruch's membrane and the retinal pigment epithelium are absent at this location. The white arrowhead points to the border of the optic nerve head. (ON = optic nerve, $V=$ vitreous; magnification as indicated in the right lower corner: black bar $100 \mu \mathrm{m}$, staining, periodic acid Schiff.) latter is histologically characterised by Bruch's membrane devoid of retinal pigment epithelium cells and photoreceptors (Fig 2). According to indirect and direct clinical histological correlations, the histological zone B corresponds to $\beta$ zone of the parapapillary chorioretinal atrophy as evaluated on ophthalmoscopy. ${ }^{917} 18$

(3) In the vicinity of zone B, zone A shows an irregular pigmentation of the retinal pigment epithelium. It correlates with $\alpha$ zone of the parapapillary atrophy. ${ }^{817}{ }^{18}$ Both zones A and B were larger in the highly myopic group than in the non-highly myopic group. This is in agreement with significantly larger $\alpha$ and $\beta$ zones in highly myopic eyes with glaucoma than in nonhighly myopic eyes with glaucoma. ${ }^{13}$

The histology of the parapapillary region explains why the blind spot is enlarged in highly myopic eyes. ${ }^{19}$ The abnormally large optic disc itself, the large peripapillary scleral ring, and the unusually large zone $\mathrm{B}$ with retinal photoreceptors missing correspond to an abnormally large absolute scotoma. ${ }^{20}$ Zone A represents a relative scotoma as shown in recent studies. ${ }^{20}$

There are factors limiting the present study. Eyes with primary open angle glaucoma were not available for histological examination. The question arises, therefore, as to how far the results of the present study can be generalised for highly myopic eyes with primary open angle glaucoma. One cannot exclude the possibility that surgical interventions such as cyclodestructive procedures may have affected the optic disc morphology. This, however, would account for both study groups since in both groups antiglaucomatous operations anterior to the ora serrata had been performed. The measurements may have been affected by swelling of the tissue after enucleation before the fixative agent reached the tissue, and the tissue dimensions may have changed by shrinkage as a result of the fixation process itself. These sources of artefact, however, affect all eyes included in the study in a similar manner and may therefore have not induced a larger error when both study groups were compared with each other. Owing to the fact that only eyes with end stage glaucoma were included, this study cannot contribute directly to an early diagnosis of glaucoma. However, the results of the study are helpful to show that in highly myopic eyes with glaucoma, the biomorphometric data of an abnormally large optic disc with shallow disc cupping and a large parapapillary atrophy agree with our histological measurements. It also shows that the whitish region at the outer border of the optic disc in highly myopic eyes with glaucoma is not parapapillary atrophy but the inner surface of the peripapillary scleral ring. This peripapillary scleral ring is covered only by the retinal nerve fibres and the inner limiting membrane (Fig 4). As in non-highly myopic eyes, it suggests that the thickness of the retinal nerve fibre layer may be measured in the region of the peripapillary scleral ring. This may be important for three dimensional evaluation of the optic nerve head. ${ }^{21}$
(2) In the parapapillary region, the peripapillary scleral ring was adjacent to zone B. The 
Supported by Deutsche Forschungsgemeinschaft (SFB 539).

1 Spaeth GL, Katz LJ, Terebuh AK. Managing glaucoma on the basis of tissue damage: a therapeutic approach based
largely on the appearance of the optic disc. In: Krieglstein largely on the appearance of the optic disc. In: Krieglstein
$\mathrm{GK}$, ed. Glaucoma update V. Heidelberg: Kaden, GK, ed. G95:118-23.

2 Jonas JB, Gusek GC, Naumann GOH. Optic disk morphometry in high myopia. Graefes Arch Clin Exp Ophthalmol 1988;226:587-90.

3 Jonas JB, Dichtl A. Optic disc morphology in the myopic primary open-angle glaucoma. Graefes Arch Clin Exp Ophthalmol 1997;(in press).

4 Wilson MR, Mortone JF. Epidemiology of chronic openangle glaucoma. In: Ritch R, Shields MB, Krupin T, eds. The glaucomas. 2nd ed. St Louis: Mosby, 1996; vol 2: chapter 35 .

5 Naumann GOH, Apple DJ. Malformations and anormalies of the eye. In: Naumann GOH, Apple DJ, eds. Pathology of the eye. Heidelberg: Springer, 1986; chapter 3.

6 Garner A. Pathology of macular degeneration in the elderly. Trans Ophthalmol Soc UK 1975;95:54-61.

7 Grossniklaus HE, Green WR. Pathologic findings in pathologic myopia. Retina 1992;12:127-33.

8 Jonas JB, Königsreuther KA, Naumann GOH. Optic disc histomorphometry in normal eyes and eyes with secondary histomorphometry in normal eyes and eyes with secondary arch Clin Exp Ophthalmol 1992;230:129-33.

9 Jonas JB, Königsreuther KA, Naumann GOH. Optic disc histomorphometry in normal eyes and eyes with secondary angle-closure glaucoma. II Parapapillary region. Graefes Arch Clin Exp Ophthalmol 1992;230:134-9.

10 Jonas JB, Zäch F-M, Gusek GC, Naumann GOH. Pseudoglaucomatous physiologic large cups. Am $\mathcal{f}$ Ophthalmol 1989;107:137-44
11 Jonas JB, Gusek GC, Naumann GOH. Optic disc, cup and neuroretinal rim size, configuration and correlations in normal eyes. Invest Ophthalmol Vis Sci 1988;29:1151-8.

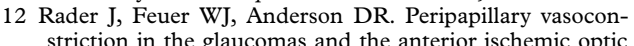
striction in the glaucomas and the anterior ischemic optic neuropathies. Am f Ophthalmol 1994;117:72-80.

13 Podos S, Becker B, Mortion W. High myopia and primary open-angle glaucoma. Am f Ophthalmol 1966;62:1039-43.

14 Perkins ES, Phelps CD. Open angle glaucoma, ocular hypertension, low-tension glaucoma, and refraction. Arch Ophthalmol 1982;100:1464-7.

15 Laatikainen L. Fluorescein angiographic studies of the peripapillary and perilimbal regions in simple, capsular and low-tension glaucoma. Acta Ophthalmol (Suppl) 1981;111: $1-83$.

16 Raitta C, Sarmela T. Fluorescein angiography of the optic disc and the peripapillary area in chronic glaucoma. Acta Ophthalmol 1970;48:303-8.

17 Fantes FE, Anderson DR. Clinical histologic correlation of human peripapillary anatomy. Ophthalmology 1989;96:205.

18 Kubota T, Jonas JB, Naumann GOH. Direct clinicohistological correlation of parapapillary chorioretinal atrophy. BrF Ophthalmol 1993;77:103-6.

19 Greve EL, Furuno F. Myopia and glaucoma. Graefes Arch Clin Exp Ophthalmol 1980;213:33-41.

20 Jonas JB, Gusek GC, Fernández MC. Correlation of the blind spot size to the area of the optic disc and barapapillary atrophy Am 7 Ophthalmol 1991;111:559-65.

21 Dichtl A, Jonas JB, Mardin CY. Comparison between laser tomographic scanning evaluation and photographic measurement of the neuroretinal rim. Am $\mathcal{f}$ Ophthalmol 1996;121:494-501. 